\title{
Phase equilibria for the oxygen-water system up to elevated temperatures and pressures
}

\author{
Xiaoyan $\mathrm{Ji}^{1,2}$, Xiaohua $\mathrm{Lu}^{2}$, Jinyue $\mathrm{Yan}^{1,3^{*}}$ \\ ${ }^{1}$ Department of Chemical Engineering and Technology / Energy Processes, \\ Royal Institute of Technology, SE -100 44 Stockholm, Sweden \\ ${ }^{2}$ Department of Chemical Engineering, Nanjing University of Chemical Technology, \\ Nanjing 210009, China \\ ${ }^{3}$ Shanghai Jiaotong University, Shanghai 200052, China
}

\begin{abstract}
A new thermodynamic model was presented to calculate the phase equilibria for the oxygen-water system. The modified RedlichKwong equation of state with a new correlated cross interaction parameter was used to calculate fugacity coefficients for the vapor phase. The dissolved oxygen followed Henry's law. A new expression was correlated from the experimental data to calculate Henry's constant of oxygen. The calculation results of equilibrium composition were compared with the available experimental data and those calculated by other models with different parameters. The comparison revealed that the new model is suitable for calculating both liquid and vapor compositions while the empirical method is only suitable for estimating the liquid composition. Furthermore, compared to the model proposed by Rebenovich and Beketov, the calculation results of the vapor composition with the new model are better.
\end{abstract}

Keywords: oxygen, water, equation of state, vapor-liquid equilibrium, Henry's constant

\section{Introduction}

Many natural and industrial processes occur in aqueous environments that involve or are affected by dissolved oxygen. Such processes range from biological and synthesis reactions to the corrosion and oxidation of materials. A detailed knowledge of temperature and pressure effects on oxygen solubility enables the related processes to be modelled more accurately and controlled more effectively. In previous literature, oxygen solubility in water was determined experimentally, and Henry's constant of oxygen in water was correlated from its partial pressure which was estimated by subtracting the vapor pressure of water from the total pressure [1-2]. Using the correlation results in the previous literature, it is reasonable to calculate the liquid composition within a narrow temperature range. However, such method is unsuitable over a wide temperature range. Furthermore, the vapor composition cannot be calculated reliably, especially at high pressures.

Meanwhile, thermodynamic properties (humidity, enthalpy and entropy) in the vapor phase for the oxygen-water system are of importance [3]. Saturated vapor

\footnotetext{
* Corresponding author, yanjy@ket.kth.se, +46 87906528
} 
composition is often needed in order to calculate saturated properties. Based on virial equation of state (EOS), Rabinovich and Beketov [3] proposed a model to calculate thermodynamic properties including saturated vapor composition for the studied system. Because of the scarcity of the experimental data, the cross interaction parameter between molecular oxygen and water was estimated from theory and the calculation results of saturated vapor composition were verified by comparing with the experimental data of the nitrogen-water system. The calculation results of the saturated composition and other properties in the vapor phase are questionable, and liquid composition cannot be calculated since the liquid phase was assumed to be pure water.

In this paper, phase equilibria of the oxygen-water system were studied. Fugacity coefficients of the components in the vapor phase were calculated with the modified Redlich-Kwong (RK) EOS [4]. The dissolved oxygen in the liquid phase followed Henry's law. Available experimental data were collected and analyzed to obtain a reasonable Henry's constant and to investigate the existing cross interaction parameter in the modified RK EOS. Calculation results in this paper were compared with experimental data and those of other models in the literature.

\section{Thermodynamic model}

The thermodynamic condition for liquid-vapor equilibrium is

$\phi_{i} y_{i} P=f_{i}^{0} x_{i} \gamma_{i}$

$\phi_{i}$ is calculated with the modified RK EOS developed by de Santis et al. [4] and

$$
\begin{aligned}
\ln \phi_{i}= & \ln \left(\frac{v}{v-b}\right)+\frac{b_{i}}{v-b}-2 \cdot \frac{\sum_{j} y_{j} a_{i j}}{R T^{1.5} b} \cdot \ln \left(\frac{v+b}{v}\right) \\
& +\frac{a b_{i}}{R T^{1.5} b^{2}} \cdot\left[\ln \left(\frac{v+b}{v}\right)-\frac{b}{v+b}\right]-\ln \left(\frac{R v}{R T}\right)
\end{aligned}
$$

where $a$ and $b$ are parameters with the mixing rule [4] and

$$
\begin{aligned}
& a=\sum_{i} \sum_{j} y_{i} y_{j} a_{i j} \\
& a_{i i}=a_{i i}^{0}+a_{i i}^{1}(T) \\
& a_{i j}=a_{j i}=\left(a_{i i}^{0} a_{j j}^{0}\right)^{0.5} \\
& b=\sum_{i} y_{i} b_{i}
\end{aligned}
$$

The temperature-independent parameters, $a_{i i}{ }^{0}$ and $b_{i}$, were taken from Wark [5]. The temperature-dependent parameter, $a_{i i}{ }^{1}$, is zero for gaseous oxygen. For water vapor, $a_{i i}{ }^{1}$ was taken from de Santis et al. [4]. Because the modified RK EOS has not been verified yet for the oxygen-water system, it was investigated in the following text.

The liquid phase reference fugacity of water $f_{w}{ }^{0}$ is calculated with equation (7). Properties of pressure and volume were calculated from the industrial standard IAPWSIF97 [6]. $\phi_{w}^{V}\left(T, P_{w}^{s}\right)$ was calculated from software of Aspen Plus with STEAMNBS model [7] and correlated with the polynomial equation (8). The sum of squares of residuals about the polynomial correlation is $1.56 \times 10^{-6}$. 


$$
\begin{aligned}
f_{w}^{0}=\phi_{w}^{V}\left(T, P_{w}^{s}\right) \cdot P_{w}^{s}(T) \cdot \exp \left[\frac{1}{R T} \int_{P_{w}^{s}}^{P} v_{w}^{L}(T, P) d P\right] \\
\begin{aligned}
\phi_{w}^{V}\left(T, P_{w}^{s}\right) & =1.30259-3.73088 \times 10^{-3} T+1.6357 \times 10^{-5} T^{2} \\
& -2.91522 \times 10^{-8} T^{3}+1.6073 \times 10^{-11} T^{4}
\end{aligned}
\end{aligned}
$$

The dissolved oxygen follows Henry's law, and $f_{i}^{0}$ of oxygen is equal to $H$ (Henry's constant). The Henry's constant of oxygen in water can be taken from literature $[1,2,8$, 9] or calculated from Helgeson EOS with equation (9) $[10,11]$ or recorrelated from the available experimental data. It was studied carefully as described as follows.

$$
H=55.51 \cdot \exp \left(\frac{\Delta G_{O_{2}, g}^{0}(T, P)_{m}-\Delta G_{O_{2}, a q}^{0}(T, P)_{m}}{R T}\right)
$$

Activity coefficients were assumed to be unity because the dissolved gas is small.

\section{Adjustment of the modified RK EOS}

For the model described above, parameters in the modified RK EOS are often correlated from the experimental data in the vapor phase. For the studied system, most of the experimental data are oxygen solubilities in the liquid phase. Although Zoss [12] determined the vapor composition up to high temperatures and pressures, it was proved that there are experimental errors [13]. Subsequently, the modified RK EOS has not been verified yet for the oxygen-water system.

Recently, Wylie and Fisher [14] determined the enhancement factor from 298.15 to $348.15 \mathrm{~K}$ and 20 to 140 bar for the oxygen-water system. Saturated vapor compositions were calculated from this group of experimental data [14] and used to investigate the modified RK EOS in this paper.

Henry's constant of oxygen in water is also needed in order to calculate the saturated vapor composition. For the first try, the Henry's constant was calculated with the Helgeson EOS from equation (9). The calculation results of water vapor composition were compared with the experimental data [14]. Comparison results are shown in Figure 1. Obviously, the higher the pressure and the lower the temperature, the higher the deviation between the calculation results and the experimental data

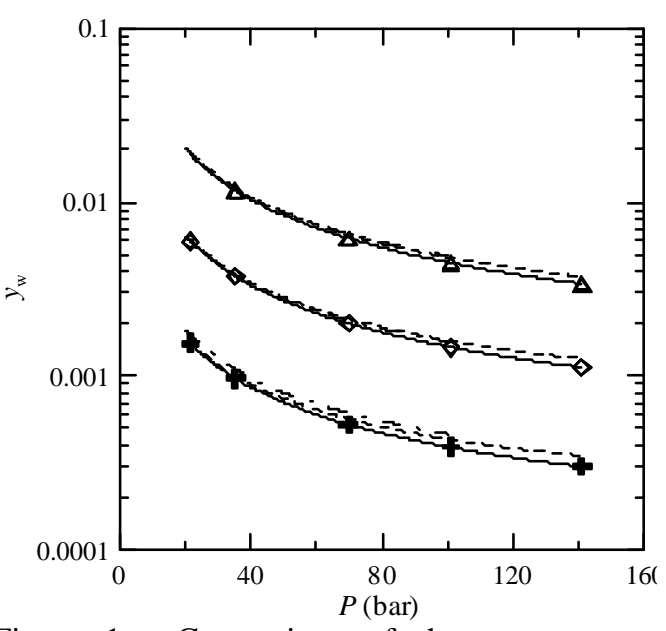

Figure 1. Comparison of the water vapor composition. $\boldsymbol{+}, \diamond$ and $\Delta$, experimental data of Wylie and Fisher [14] at 298.15, 323.15 and $348.15 \mathrm{~K}$, respectively. --- and - calculation results of the modified RK EOS with the original and new correlated parameters, respectively; -.., calculation results of Rabinovich and Beketov [3]. of Wylie and Fisher [14]. The deviation is about 15\% at $298.15 \mathrm{~K}$ and 140.85 bar.

The deviation may be due to two factors, the parameters in the modified RK EOS and the Henry's constant calculated with the Helgeson EOS. At low temperature $(<100$ ${ }^{\circ} \mathrm{C}$ ), parameters in the Helgeson EOS were correlated from experimental data of Benson 
et al. [8] in which the Henry's constant of oxygen in water was correlated from the accurate oxygen solubility with a semi-empirical method. Therefore, we can conclude that the Henry's constant calculated with the Helgeson EOS should be correct in the low temperature range. Wylie and Fisher [14] also stated that the effect of oxygen on the concentration of water vapor with liquid water is mainly caused by intermolecular forces in the gas phase. The Poynting effect is about one third compared to intermolecular forces of gas, and the Raoult effect is much less compared to intermolecular forces of gas and the Poynting effect. In other words, the effect of Henry's constant on the vapor composition is small. Therefore, the deviation is due to the parameters in the modified RK EOS.

In this paper, an adjustable parameter $k$ was added in order to calculate the cross interaction parameter as shown in equation (10). $k$ was correlated from experimental data of Wylie and Fisher [14]. The correlation result is also shown in equation (10).

$$
a_{i j}=a_{j i}=k \cdot\left(a_{i i}^{0} a_{j j}^{0}\right)^{0.5}=0.783 \cdot\left(a_{i i}^{0} a_{j j}^{0}\right)^{0.5}
$$

\section{Correlation of the Henry's constant of oxygen in water}

\section{Correlation for each experimental data point}

The Henry's constant of oxygen in water can be correlated from solubility data which have been determined experimentally [1, 8, 9, 12, 13, 15-18]. In most of these studies, the partial pressure of oxygen was reported which was estimated by subtracting the vapor pressure of water from the total pressure. When a gas dissolves in water, the partial pressure of water will deviate from the saturated pressure of pure water, especially at high pressure. It indicates that the estimated partial pressure of oxygen in the literature is unreasonable.

In this paper, the Henry's constant of oxygen in water was correlated from the solubility data at the experimental temperature and total pressure. However, in the work of Cramer [1], the total pressure was not reported, thus we have not included the data of Cramer [1] in our correlation. It was proved that the solubility values of Zoss [12] are more than an order of magnitude high at low temperature, therefore, they were not considered in the correlation and their experimental errors were checked in the later text. The data of Stephan et al. [9] covered the data of Pray and Stephan [17], so the data of Pray and Stephan [17] were excluded in the correlation. The data of Rettich et al. [18] were also not considered because the temperature range is within that of Benson et al. [8], and the deviation between them is small.

Henry's constant was correlated for each experimental data point with the Newton's method. However, for the data of Benson et al. [8], Henry's constant for each experimental data point has been correlated, and it was used directly in this paper.

\section{Correlation of the expression of $H=f(T, P)$}

An appropriate expression, equation (11), was chosen for the expression of $H$. The errors of the available experimental data have not been estimated in previous studies. In order to obtain reasonable correlation results, each experimental data point has an equivalent weight in our correlation. But in this case a large error in one group will cause a large deviation in the correlation. In order to avoid this, each group of data were 
correlated first with equation (11). Since the number of the data of Pray et al. [16] is ess than that of parameters in the correlation expression, the data cannot be checked in this step. The correlation results for other groups are listed in Table 1.

$$
\begin{aligned}
& \ln H=Q_{1}+Q_{2} P+Q_{3} P^{2}+\left(Q_{4}+Q_{5} P+Q_{6} P^{2}\right) \cdot T+ \\
& \quad\left(Q_{7}+Q_{8} P+Q_{9} P^{2}\right) \cdot T^{2}+\left(Q_{10}+Q_{11} P+Q_{12} P^{2}\right) \cdot \ln T
\end{aligned}
$$

Table 1. Correlation results for each group of data and multi-groups of data

\begin{tabular}{ccc}
\hline No & data source & R-squared \\
\hline 1 & Benson et al. [8] & $>0.9999$ \\
2 & Stephan et al. [9] & 0.9972 \\
3 & Brodén and Simonson [15] & 0.9999 \\
4 & case A & 0.9917 \\
5 & case B & 0.9970 \\
\hline
\end{tabular}

case A, four groups of data (Benson et al. [8]; Stephan et al. [9] Brodén and Simonson [15]; Pray et al. [16]) were used in the correlation.

case B, three groups of data (Benson et al. [8] ; Stephan et al. [9]; Brodén and Simonson [15]) were used in the correlation

As shown in Table 1, the random experimental error for each group of data is small (No. 1 to No. 3). Therefore, all of them combined with the data of Pray et al. [16] (case A) were used to correlate the parameters in equation (11). The correlation results are listed in Table 1 (No. 4) and Figure 2. From the correlation results shown in Figure 2, it is clear that some experimental data caused large deviations. The data of Pray et al. [16] may be responsible because they had not been checked separately before correlation. In order to verify this conclusion, three groups of data $[8,9,15]$ (case B) were used to correlate the parameters in equation (11). The correlation results are also listed in Table 1 (No. 5) and shown in Figure 3. The value of R-squared of case B is near to that of case $\mathrm{A}$, but the distribution of the deviation of case B is much better than that of case A as shown in Figures 2 and 3. Therefore, the correlation results of case B are reasonable and the corresponding correlation results for each parameter are listed in Table 2.

Table 2. Coefficients in equation (11) for calculating the Henry's constant of oxygen

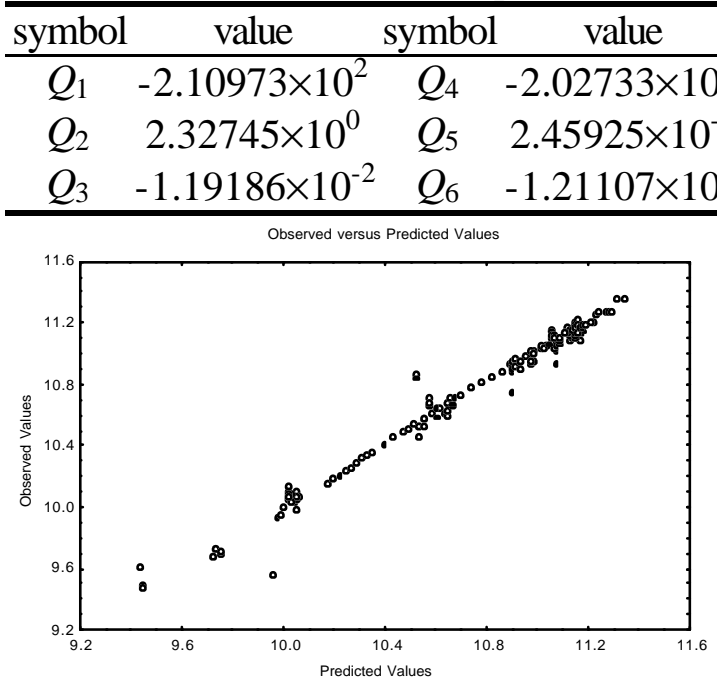

Figure 2. Observed versus predicted values $(\ln \mathrm{H})$ with equation (11) when four groups of data (case A) were used to correlate the expression.

symbol value symbol value

$\begin{array}{llll}Q_{7} & 9.77301 \times 10^{-5} & Q_{10} & 4.79875 \times 10^{1}\end{array}$

$Q_{8} \quad-1.43857 \times 10^{-6} \quad Q_{11} \quad-5.14296 \times 10^{-1}$

$\begin{array}{llll}Q_{9} & 6.84983 \times 10^{-9} & Q_{12} & 2.61610 \times 10^{-3}\end{array}$

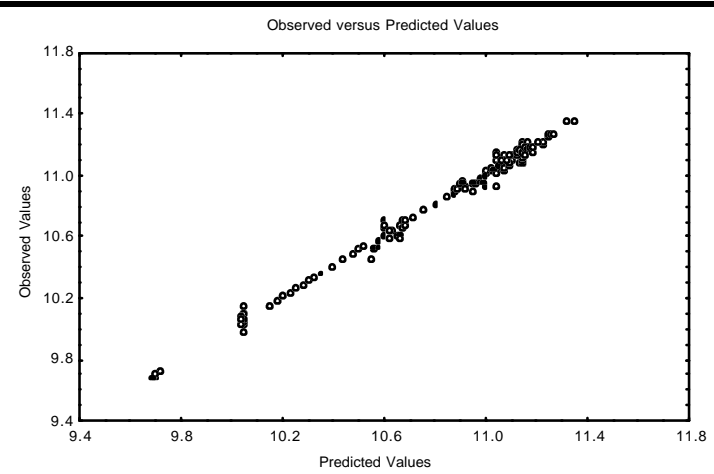

Figure 3. Observed versus predicted values $(\ln \mathrm{H})$ with equation (11) when three groups of data (case B) were used to correlate the expression. 


\section{Comparison and discussion}

\section{Direct comparison of the Henry's constant}

In the paper of Benson et al. [8], the Henry's constant was correlated at the determined total pressure which is below 1.2 bar. In the studies of Cramer [1] and Tromans [2], the Henry's constant was correlated at high temperature. These correlation results were compared with those in this paper. From the comparison results shown in Figure 4, it is clear that the correlation results in this paper are consistent with those in the literature. In other words, when the pressure is near or lower than the saturated pressure of water, its effect on Henry's constant can be neglected.

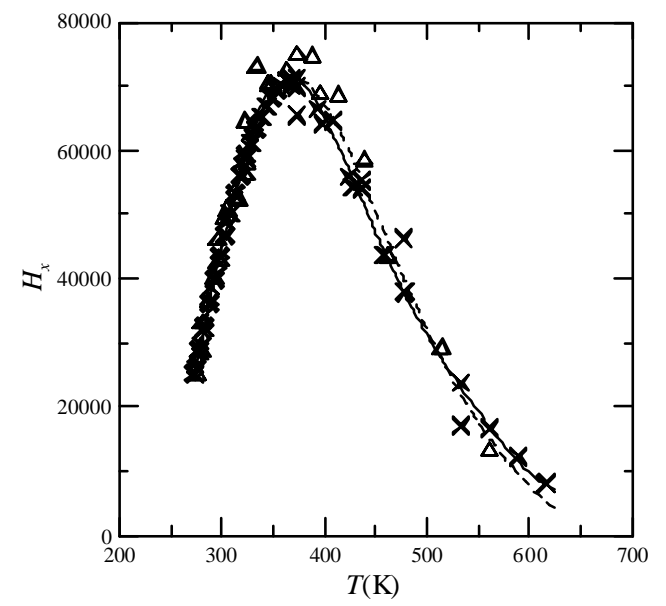

Figure 4. Comparison of Henry's constant at the saturated pressure of water. $\Delta, \times$ and $\diamond$, experimental data of Cramer [1], Tromans [2] and Benson et al. [8], respectively. - , correlation results in this paper. --- , calculation results from the Helgeson EOS.

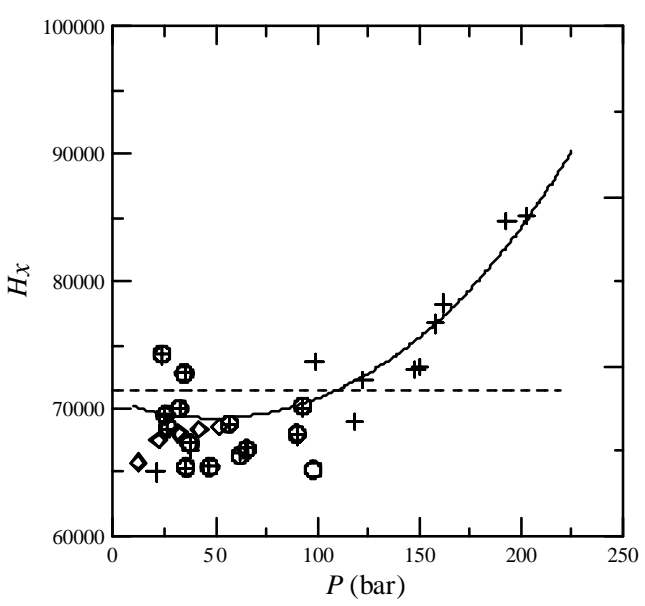

Figure 5. Comparison of Henry's constant at $373.15 \mathrm{~K} .+\boldsymbol{+}, \diamond$ and $\bigcirc$, experimental data of Stephan et al [9], Brodén and Simonson [15] and Pray and Stephan [17], respectively. - , correlation results of this paper; -.--, correlation results of Tromans [2].

Figure 5 shows the pressure effect on Henry's constant at $373.15 \mathrm{~K}$. At pressure below 150 bar, the effect of pressure on Henry's constant can be neglected. When the pressure is high, the value of Henry's constant increases obviously. In this case, it is necessary to consider the pressure impact in order to obtain reliable results. Correlation results of Tromans [2] are also plotted as a dashed line in Figure 5. Obviously, the results in this paper are much better than those of Tromans [2] at high pressure.

In the former text, the Henry's constant of oxygen in water was calculated first from Helgeson EOS with equation (9) in order to correlate the cross interaction parameter in the modified RK EOS. Therefore, it is necessary to check the calculation results of Helgeson EOS. The Henry's constant at the saturated pressure of water was calculated with equation (9) and compared with those correlated in this paper. The comparison results are depicted in Figure 4 as a dashed line. At low temperature $(<400$ $\mathrm{K}$ ), results from Helgeson EOS are very close to those correlated in this paper since these two studies are all based on the results of Benson et al. [8] at low temperature. This also implies that the correlation of the cross interaction parameter for the oxygenwater system in the modified RK EOS is reasonable.

When the temperature is high, there is some deviation between the calculation results of this paper and those calculated with the Helgeson EOS. This is explained by 
the following text. In the Helgeson EOS [10, 11], the parameter of the effective Born coefficient $\left(w_{\mathrm{e}}\right)$ for the neutral species $\left(\mathrm{O}_{2}\right.$,aq $)$ was correlated from the equilibrium constant. The equilibrium constant was calculated from the solubility data in which the partial pressure was estimated by subtracting vapor pressure of water from total pressure [10]. The deviation of the estimated partial pressure caused the deviation in the Henry's constant. This also indicates that it is necessary to make a further study on how to improve and correct the parameter of the Helgeson EOS.

\section{Comparison of the composition}

Composition was calculated here with two methods, thermodynamic and empirical ones, in order to understand the effects of Henry's constant and the method on the equilibrium composition. In the thermodynamic method, the partial pressure of water was calculated when each component satisfied the conditions for phase equilibria. In the empirical method, the partial pressure of water is the saturated pressure of pure water.

In both of these two methods, the Henry's constant of oxygen in water was needed. The Helgeson EOS [10,11] can be used to calculate the Henry's constant at a certain temperature and pressure. Tromans [2] correlated the Henry's constant from 273 to 613 $\mathrm{K}$ and the partial pressure up to 60 bar. In this paper, a new expression for Henry's constant was correlated. Therefore, the Henry's constant was calculated or taken from these three studies respectively to calculate the composition for comparison.

In addition, in the thermodynamic method, fugacity coefficient in the vapor phase was calculated by the modified RK EOS with the existing parameter and the new parameter correlated in this paper, respectively.

Based on the above discussion, five cases were calculated in this paper:

- Case 1, thermodynamic method, the Henry's constant was calculated from the correlation expression in this paper and the fugacity coefficients were calculated by the modified RK EOS with the new parameter correlated in this paper.

- Case 2, thermodynamic method, the Henry's constant was calculated from the Helgeson EOS and the fugacity coefficients were calculated by the modified RK EOS with the new parameter correlated in this paper.

- Case 3, thermodynamic method, the Henry's constant was calculated from the Helgeson EOS and the fugacity coefficients were calculated from the modified RK EOS with the existing parameter in the literature [10].

- Case 4, empirical method, $x_{\mathrm{O} 2}=P_{\mathrm{O} 2} \phi_{\mathrm{O} 2}(T, P) / H, y_{w}=P_{w}^{s} / P$. $\phi_{\mathrm{O} 2}$ was calculated by the modified RK EOS with the existing parameter. The Henry's constant was calculated from the Helgeson EOS.

- Case 5, empirical method, $x_{\mathrm{O} 2}=P_{\mathrm{O} 2} / H, y_{w}=P_{w}^{s} / P$. The Henry's constant was taken from Tromans [2].

Calculation results at $560.93 \mathrm{~K}$ and different pressures were shown as an example. The calculation results of the oxygen solubility in water $\left(x_{\mathrm{O} 2}\right)$ were compared with experimental data of Stephan et al. [9]. However, experimental data in the vapor phase have not been determined at this temperature. In order to show the difference between different methods, the calculation results of this paper (Case 1) were used as reference 
data because their accuracy has been checked in the previous text. The calculation and comparison results are shown in Table 3.

Table 3. Equilibrium composition for the oxygen (1) -water (2) system at $560.93 \mathrm{~K}^{*}$ calculated with different methods and parameters

\begin{tabular}{|c|c|c|c|c|c|c|c|c|c|c|c|}
\hline \multirow{3}{*}{\multicolumn{2}{|c|}{$P$ (bar) $10^{4} x_{1 \exp }$}} & \multicolumn{6}{|c|}{ Thermodynamic method } & \multicolumn{4}{|c|}{ Empirical method } \\
\hline & & \multicolumn{2}{|c|}{ Case 1} & \multicolumn{2}{|c|}{ Case 2} & \multicolumn{2}{|c|}{ Case 3} & \multicolumn{2}{|c|}{ Case $4 * *$} & \multicolumn{2}{|c|}{ Case 5} \\
\hline & & $10^{4} x_{1}$ & $y_{2 r}$ & $10^{4} x_{1}$ & $y_{2}$ & $10^{4} x_{1}$ & $y_{2}$ & $10^{4} x_{1}$ & $y_{2}$ & $10^{4} x_{1}$ & $y_{2}$ \\
\hline 103.7 & 8.4 & 18.22 & & & & 96 & 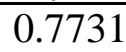 & & 0. & 3.78 & 0. \\
\hline 105 & 19.5 & 19.42 & 0.7606 & 20.49 & 0.7605 & 20.08 & 0.7 & 2 & 0.6 & 19.98 & 0.6 \\
\hline 13 & 38 & 37 & 0. & 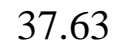 & 0.6 & 36 & 0. & & & 37 & \\
\hline & 39.5 & 1 & & & 0. & 38 & & & & 39.77 & \\
\hline 17 & 61.2 & 96 & & & 0 & 72 & 0 . & 57 & & 59.15 & 0.4 \\
\hline 172.6 & 61.9 & 60.85 & 0.5231 & 57.73 & 0.5234 & 56.25 & 0.5336 & 60.42 & 0.4189 & 59.98 & 0.4189 \\
\hline \multicolumn{2}{|c|}{ Max. (\%) } & 2.57 & - & 7. & 0.06 & 9.13 & 2.0 & 10.51 & 19.92 & 3.34 & 19.92 \\
\hline \multicolumn{2}{|c|}{ Av. (\%) } & 1.33 & - & 4.33 & 0.02 & 5.07 & 1.09 & 4.07 & 15.10 & 2.27 & 15.10 \\
\hline
\end{tabular}

* The saturated pressure $P_{w}^{s}$ is 73.3 bar at $560.93 \mathrm{~K}$.

$* *$ If the second point was excluded, Max. $=4.81 \%, \mathrm{Av}=2.79 \%$.

$$
\begin{aligned}
& \text { Max. }=\max \left(\frac{\left|x_{1}-x_{1 \exp }\right|}{x_{1 \exp }}\right)_{i} \text { or }=\max \left(\frac{\left|y_{2}-y_{2 r}\right|}{y_{2 r}}\right)_{i} \quad(i=1,6) \\
& \text { Av. }=\frac{1}{6} \sum_{i=1}^{6}\left(\frac{\left|x_{1}-x_{1 \exp }\right|}{x_{1 \exp }}\right)_{i} \text { or } \quad=\frac{1}{6} \sum_{i=1}^{6}\left(\frac{\left|y_{2}-y_{2 r}\right|}{y_{2 r}}\right)_{i}
\end{aligned}
$$

In cases 1 and 2, the Henry's constant was taken from different studies. The water vapor in different cases is nearly the same. The maximum deviation is about $0.06 \%$. However, the calculation results of oxygen solubilities in case 1 are much better than those in case 2 by comparing with the experimental data. This implies that the effect of the Henry's constant on liquid composition is obvious while its effect on vapor composition is small enough to be neglected.

In cases 2 and 3, fugacity coefficients were calculated from the modified RK EOS with the new correlated and existing parameters, respectively. Comparing the calculation results of these two cases, one conclusion can be drawn. The effects of the parameter in the modified RK EOS on liquid and vapor compositions are visible.

In cases 4 and 5 , the composition of water vapor was calculated by the empirical method. Comparing the calculation results of water vapor in these two cases with those in case 1, the deviation is large and the maximum value is up to $20 \%$. However, the calculation results of liquid composition in these two cases are better than those in case 3. It implies that the empirical method is suitable for calculating the liquid composition.

Based on the above comparison, we can conclude that the thermodynamic method is better than the empirical method. Furthermore, with the new parameter in the modified RK EOS and the Henry's constant correlated in this paper, the calculation results for both liquid and vapor compositions are better than other models. 


\section{Comparison with the model proposed by Rabinovich and Beketov [3]}

Rabinovich and Beketov [3] calculated and tabulated saturated vapor composition from 200 to $400 \mathrm{~K}$ and 1 to $100 \mathrm{bar}$. In order to investigate the calculation results of this model, data at $298.15,323.15$ and $348.15 \mathrm{~K}$ were interpolated from 20 to 100 bar and compared with the experimental data of Wylie and Fisher [14]. The comparison results are shown in Figure 1. The calculation results of Rabinovich and Beketov [3] are higher than the experimental data of Wylie and Fisher [14]. The lower the temperature and the higher the pressure, the lager the deviation.

The calculation results of the new model with the new correlated parameters are also shown in Figure 1 for comparison. From 298.15 to $348.15 \mathrm{~K}$, the average deviation of the calculation results in this paper from the experimental data [14] is within $0.3 \%$, and the maximum deviation is only $0.5 \%$. It implies that the correlation is reasonable, and the calculation results of this paper are much better than those of Rabinovich and Beketov [3].

\section{Investigation of the data of Zoss [12]}

Vapor and liquid compositions were determined experimentally by Zoss [12]. However, it was stated that the data of liquid composition are more than an order of magnitude high at low temperature. In the literature [13], it was also proved that the solubility data at the higher temperatures appears to be reliable. We made a comparison of the calculation results with the data of Zoss [12]. Figure 6 gives the comparison results for the liquid composition. At high temperatures and pressures, the calculation results agree with the experimental data of Zoss [12]. Therefore, the calculation results are also

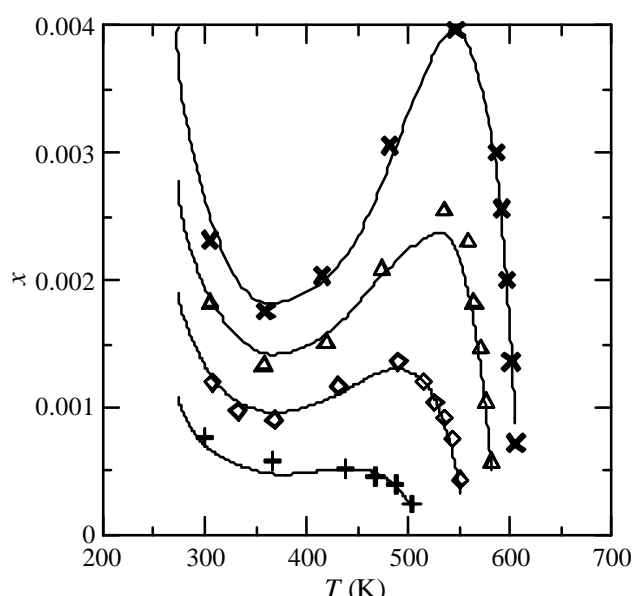

Figure 6. Comparison of the calcualtion results of liquid composition of water with experimental data of Zoss [10]. $\boldsymbol{+}, \diamond, \Delta$, and $\mathbf{X}$, experimental data at $34.5,68.95,103.4$ and 137.9 bar, respectively; - calculation results in this paper. accurate enough up to $605 \mathrm{~K}$.

Table 4. Comparison of the calcualtion results of water vapor composition $\left(y_{\mathrm{wcal}}\right)$ in this paper with the experimental data of Zoss [12] $\left(y_{\text {wexp }}\right)$

\begin{tabular}{ccccccccc}
\hline \multicolumn{3}{c}{ 68.95 bar } & \multicolumn{3}{c}{103.4 bar } & \multicolumn{3}{c}{137.9 bar } \\
\hline$T(\mathrm{~K})$ & $y_{\text {wexp }}$ & $y_{\text {wcal }}$ & $T(\mathrm{~K})$ & $y_{\text {wexp }}$ & $y_{\text {wcal }}$ & $T(\mathrm{~K})$ & $y_{\text {wexp }}$ & $y_{\text {wcal }}$ \\
\hline 307.0 & 0.137 & $8.81 \mathrm{E}-4$ & 305.4 & 0.273 & $5.76 \mathrm{E}-4$ & 304.3 & 0.155 & $4.35 \mathrm{E}-4$ \\
355.9 & 0.133 & $8.55 \mathrm{E}-3$ & 360.4 & 0.248 & $7.12 \mathrm{E}-3$ & 358.2 & 0.090 & $5.13 \mathrm{E}-3$ \\
369.8 & 0.203 & $1.45 \mathrm{E}-2$ & 417.6 & 0.427 & $4.54 \mathrm{E}-2$ & 413.7 & 0.338 & $3.16 \mathrm{E}-2$ \\
429.3 & 0.594 & $9.00 \mathrm{E}-2$ & 472.0 & 0.731 & $1.70 \mathrm{E}-1$ & 484.8 & 0.719 & $1.72 \mathrm{E}-1$ \\
489.3 & 0.882 & $3.55 \mathrm{E}-1$ & 534.8 & 0.917 & $5.41 \mathrm{E}-1$ & 547.6 & 0.910 & $5.18 \mathrm{E}-1$ \\
\hline
\end{tabular}

Table 4 shows the comparison of the water vapor composition calculated in this paper with the experimental data of Zoss [12]. The experimental data of Zoss [12] are much higher than the calculation results in this paper. Because our calculation results 
have been evaluated by the experimental data of Wylie and Fisher [14] from 298.15 to $348.15 \mathrm{~K}$ and $140 \mathrm{bar}$, there might be some errors in the experimental data of Zoss [12].

\section{Conclusions}

Phase equilibria for the oxygen-water system were studied by the thermodynamic method in which the modified Redlich-Kwong EOS with a new correlated parameter was used to calculate fugacity coefficients for the vapor phase and the dissolved gas followed Henry's law. A reliable expression was obtained to calculate fugacity coefficient of pure saturated water accurately, and a new Henry's constant of oxygen in water was correlated from the experimental data. Furthermore, the investigation of the Henry's constant of oxygen in water calculated with the Helgeson EOS shows that there are some deviations at high temperatures and pressures, which means that new parameters in Helgeson EOS are to be re-correlated.

Equilibrium compositions were calculated by the new model with the parameters suggested in this paper and compared with experimental data and those calculated by other models with different parameters. The comparison revealed that the new model is reliable for calculating liquid and vapor compositions, and the calculation results of the vapor composition are much better than those in the study of Rabinovich and Beketov [3], while the empirical method is only suitable for estimating the liquid composition. Meanwhile, there might be some errors in the experimental data of Zoss [12].

\section{List of symbols}

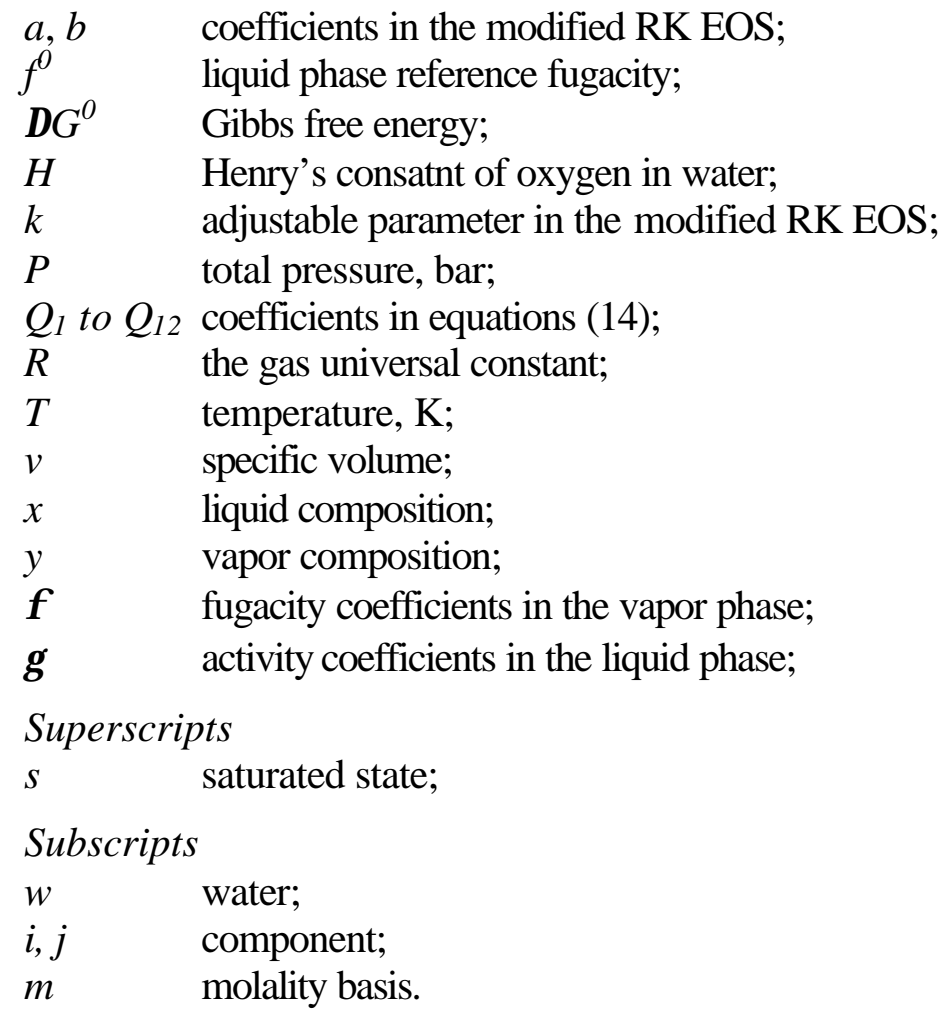




\section{Acknowledgement}

Financial support from the Swedish Energy Agency and the outstanding youth of National Nature Science Foundation of China (29925616) is gratefully acknowledged.

\section{References}

[1] S. D. Cramer, Ind. Eng. Chem. Process Des. Dev., 19 (1980) 300-305.

[2] D. Tromans, Hydrometallurgy, 48 (1998) 327-342.

[3] V. A. Rabinovich, and V. G. Beketov, Moist gases, thermodynamic properties, Begell: House, 1995

[4] R. de Santis, G. J. F. Breedveld and J. M. Prausnitz, Ind. Eng. Chem., Process Des. Devlop., 13 (1974) 374-377.

[5] K. Wark, Advanced thermodynamics for engineers, McGraw-Hill, Inc., Singapore, 1995.

[6] W. Wagner and A. Kruse, Properties of water and steam, Springer, New York, 1998.

[7] Aspen Plus, UserGuide, Aspen Tech. Inc., Cambridge, MA, 1999

[8] B. B. Benson, D. Krause and M. A. Peterson, J. Solu. Chem., 8 (1979) 655-690.

[9] E. F. Stephan, N. S. Hatfield, R. S. Peoples and H. A. H. Pray, The solubility of gases in water and in aqueous uranyl solutions at elevated temperature and pressure, BMI, 1067, 54, Battelle Mem. Inst., Columbus, OH., 1956.

[10] E. L. Shock, H. C. Helgeson and D. A. Sverjensky, Geochimica \& Cosmochimica Acta, 53 (1989) 2157-2183.

[11] J. W. Johnson, E. T. Oelkers and H. C. Helgeson, Computers \& Geosciences, 18 (1992) 899-947.

[12] L. M. Zoss, 1952, cited in Battino (1981).

[13] R. Battino, Oxygen and Ozoner, IUPAC solubility data series 7, Pergamon Press, Oxford, 1981.

[14] R. G. Wylie and R. S. Fisher, J. Chem. Eng. Data, 41 (1996), 175-180.

[15] A. Brodén and R. Simonson, Svensk Parrerstidning, 81 (1978), 541-544.

[16] H. A. Pray, C. E. Schweickert and B. H. Minnich, Ind. Eng. Chem., 44 (1952), 1146-1151.

[17] H. A. Pray and E. F. Stephan, 1953, cited in Battino (1981).

[18] T. R. Rettich, R. Battino and E. Wilhelm, J. Chem. Thermodyn., 32 (2000) 1145-1156. 